\title{
Particle Learning Methods for State and Parameter Estimation
}

\author{
Christopher Nemeth ${ }^{1}$, Paul Fearnhead ${ }^{1}$, Lyudmila Mihaylova ${ }^{2}$ and Dave Vorley ${ }^{3}$ \\ ${ }^{1}$ Dept. of Mathematics and Statistics, Lancaster University, UK \\ ${ }^{2}$ School of Computing and Communications, Lancaster University, UK \\ ${ }^{3}$ MBDA, Filton, Bristol, UK \\ \{c.nemeth,p.fearnhead,mila.mihaylova\}@lancaster.ac.uk,dave.vorley@mbda-systems.com
}

\begin{abstract}
This paper presents an approach for online parameter estimation within particle filters. Current research has mainly been focused towards the estimation of static parameters. However, in scenarios of target maneuverability, it is often necessary to update the parameters of the model to meet the changing conditions of the target. The novel aspect of the proposed approach lies in the estimation of non-static parameters which change at some unknown point in time. Our parameter estimation is updated using changepoint analysis, where a changepoint is identified when a significant change occurs in the observations of the system, such as changes in direction or velocity.
\end{abstract}

Keywords - parameter estimation, Monte Carlo methods, nonlinear filtering, changepoint detection

\section{Introduction}

Nonlinear filtering problems can be challenging as the optimal filter does not admit a closed-form expression. In target tracking, this is a common problem when tracking an object using nonlinear measurements such as range and bearings. Sequential Monte Carlo (SMC) methods, also known as particle filters, approximate the posterior distribution of the filtered states with a discrete set of samples/particles. These techniques have been shown to provide improved estimates over deterministic approximation approaches (e.g. extended Kalman filter) when the state-space model is nonlinear and/or non-Gaussian.

In standard filtering problems it is often assumed that the parameters of the model are known. However, in recent years, developments have been made to address the issue of parameter estimation within particle filters (see [1] for a review of parameter estimation techniques). The majority of work in this area has been focused on the estimation of unknown static parameters with little work relating to the problem of estimating time-varying parameters. We consider piecewise time-varying parameters, where at some unknown time points the parameters switch to new parameter values. However, between switching periods the parameters are treated as static and estimated using methods for the estimation of static parameters. This problem is of particular interest in target tracking where a target may switch between periods of high and low maneuverability.

A popular alternative to estimating piecewise time-varying parameters is to use filters which combine multiple potential models such as the interacting multiple model (IMM) filter [2]. In this types of filter, numerous models are proposed (e.g. models for constant velocity and coordinated turn), each of which permit different fixed parameters, allowing the filter to switch between models depending on the motion of the target. The IMM filter has proven to be very successful for tracking highly maneuverable targets. However, the reliability of the IMM filter is dependent on the number and choice of models which must be pre-specified before the filter is intialised.

In this paper we propose an approach which allows for the online estimation of piecewise timevarying parameters. This work is based on the recent particle learning approach [3] to parameter estimation which relies on low-dimensional sufficient statistics for the estimation of unknown static parameters. Our approach extends this technique to account for the time-varying case by including changepoint analysis to identify points in time where the motion of the target has changed significantly. By including this changepoint approach to parameter estimation the filter is able to adapt the parameters of the model to match the changing conditions of the target.

This paper is structured as follows. Section 2 presents the state-space model and introduces standard particle filtering. In Section 3 particle filtering is extended to include our approach and alternative approaches which allow for online estimation of parameters, in conjunction with state estimation. Section 4 compares the performance of the algorithms in Section 3 using a target tracking example. Finally, conclusions are given in Section 5 . 


\section{State-Space Models and Particle Filtering}

The target tracking problem can be formulated within the framework of a state-space model. Let $\mathbf{X}_{t}$ and $\mathbf{Z}_{t}$ be two stochastic processes that are dependent on parameters $\boldsymbol{\theta}$ (n.b. $\boldsymbol{\theta}$ relates to static parameters and $\boldsymbol{\theta}_{t}$ relates to piecewise time-varying parameters). The process $\mathbf{X}_{t}$ represents the hidden state of a target at time $t$ and $\mathbf{Z}_{t}$ is the observation process where the observations are assumed to be conditionally independent given $\mathbf{X}_{t}$, with initial density $p\left(\mathbf{x}_{1} \mid \boldsymbol{\theta}\right)$. The state and observation processes are given according to probability densities

$$
\begin{aligned}
& \mathbf{X}_{t} \mid \mathbf{X}_{t-1}=\mathbf{x}_{t-1} \sim p\left(\mathbf{x}_{t} \mid \mathbf{x}_{t-1}, \boldsymbol{\theta}\right) \\
& \mathbf{Z}_{t} \mid \mathbf{X}_{t}=\mathbf{x}_{t} \sim p\left(\mathbf{z}_{t} \mid \mathbf{x}_{t}, \boldsymbol{\theta}\right)
\end{aligned}
$$

where the state model is Markov, conditional on the previous state and parameters $\boldsymbol{\theta}$ with the observations at time $t$ conditional on the state at time $t$.

Determining the underlying state at any given time requires the filtered posterior distribution $p\left(\mathbf{x}_{t} \mid \mathbf{z}_{1: t}, \boldsymbol{\theta}\right)$ to be estimated recursively in time. The posterior distribution summarises the information about the state of the target $\mathbf{x}_{t}$ from the observations $\mathbf{z}_{1: t}$, where $\mathbf{z}_{1: t}=\left\{\mathbf{z}_{1}, \mathbf{z}_{2}, \ldots, \mathbf{z}_{t}\right\}$. The posterior distribution of the filtered states can be derived recursively using Bayes rule

$$
p\left(\mathbf{x}_{t}, \boldsymbol{\theta} \mid \mathbf{z}_{1: t}\right)=\frac{p\left(\mathbf{z}_{t} \mid \mathbf{x}_{t}, \boldsymbol{\theta}\right) p\left(\mathbf{x}_{t}, \boldsymbol{\theta} \mid \mathbf{z}_{1: t-1}\right)}{p\left(\mathbf{z}_{t} \mid \mathbf{z}_{1: t-1}, \boldsymbol{\theta}\right)}
$$

where,

$$
p\left(\mathbf{x}_{t}, \boldsymbol{\theta} \mid \mathbf{z}_{1: t-1}\right)=\int p\left(\mathbf{x}_{t} \mid \mathbf{x}_{t-1}, \boldsymbol{\theta}\right) p\left(\mathbf{x}_{t-1}, \boldsymbol{\theta} \mid \mathbf{z}_{1: t-1}\right) d \mathbf{x}_{t-1}
$$

and

$$
p\left(\mathbf{z}_{t} \mid \mathbf{z}_{1: t-1}, \boldsymbol{\theta}\right)=\int p\left(\mathbf{z}_{t} \mid \mathbf{x}_{t}, \boldsymbol{\theta}\right) p\left(\mathbf{x}_{t}, \boldsymbol{\theta} \mid \mathbf{z}_{1: t-1}\right) d \mathbf{x}_{t} .
$$

Evaluating the posterior distribution (1) analytically is only possible when either the state-space is finite or linear-Gaussian. In scenarios where this is not the case it is possible to approximate the posterior distribution using a discrete set of random samples/particles $\left\{\mathbf{x}_{t}^{(i)}\right\}_{i=1}^{N}$ with associated weights $\left\{w_{t}^{(i)}\right\}_{i=1}^{N}$. An approximation of the posterior distribution is then characterised as

$$
p\left(\mathbf{x}_{t}, \boldsymbol{\theta} \mid \mathbf{z}_{1: t}\right) \approx \sum_{i=1}^{N} w_{t}^{(i)} \delta_{\mathbf{x}_{t}^{(i)}}\left(\mathbf{x}_{t}\right)
$$

where $\delta(\cdot)$ is the Dirac delta function and each particle $\mathbf{x}_{t}^{(i)}$ has weight $w_{t}^{(i)}$ and $N$ is the number of particles.
The particle approximation of the posterior distribution is updated recursively by propagating and updating the particles according to the system dynamics. Usually a resampling step is included, where the particles are resampled so as to duplicate particles with high weights and remove particles which carry low weights. This step acts to reduce the degeneracy of the particle approximation, which after a few iterations, can lead to a pointmass representation of the posterior, as all but one particle will carry a non-negligible weight.

\section{Parameter Estimation}

The problem of parameter estimation within particle filters has long been considered within two frameworks, online and offline. Here we will focus solely on parameter estimation in an online setting where the aim is to estimate unknown parameters in conjunction with estimating the state. Early approaches to this problem involved treating the parameters as an extension of the state and then solving the problem with standard particle filter methods. However, as the parameters are static, resampling $\boldsymbol{\theta}$ over time will lead to a point-mass approximation of the marginal posterior distribution for the parameters [4]. Gordon et al. [5] suggest adding artificial noise to the parameters to reduce the problem of particle depletion. However, this approach leads to a distortion of the parameters' distribution as adding artificial noise to the parameters will lead to diffuse marginal posterior distributions for the parameters. Popular recent alternatives have included Markov chain Monte Carlo (MCMC) steps within particle filters to estimate the unknown parameter [6]. However, such approaches tend to reduce the speed on the filter and can make the approach impractical within an online setting.

\subsection{Particle Learning}

Carvalho et al. [3] have recently presented an approach to online parameter estimation which combines the auxiliary particle filter [7] and the Storvik filter [8] for parameter estimation. The auxiliary particle filter weights particles according to the predictive likelihood $p\left(\mathbf{z}_{t} \mid \mathbf{x}_{t-1}, \boldsymbol{\theta}\right)$, this has been found to provide better estimates as the propagation of particles takes account of the newest observation $\mathbf{z}_{t}$. However, it is generally not possible to evaluate $p\left(\mathbf{z}_{t} \mid \mathbf{x}_{t-1}, \boldsymbol{\theta}\right)$, instead, an estimate $\boldsymbol{\mu}_{t}$ of the transition density $p\left(\mathbf{x}_{t} \mid \mathbf{x}_{t-1}, \boldsymbol{\theta}\right)$ is used to give $p\left(\mathbf{z}_{t} \mid \boldsymbol{\mu}_{t}, \boldsymbol{\theta}\right)$. This estimate is usually the mean, mode or median of the transition density. Here we will use

$$
\boldsymbol{\mu}_{t}^{(i)}=\mathrm{E}\left(\mathbf{x}_{t} \mid \mathbf{x}_{t-1}^{(i)}, \boldsymbol{\theta}^{(i)}\right)
$$

as a prior point mass estimate of $\left(\mathbf{x}_{t-1}, \boldsymbol{\theta}\right)$, where $\mathrm{E}(\cdot)$ is the mathematical expectation operator. 
Particle learning has been found to outperform the Storvik filter and perform comparably against MCMC samplers [3], particularly over long periods of time. One of the unique features of this filter is the use of sufficient statistics for state and parameter estimation. Simulation from $p\left(\boldsymbol{\theta} \mid \mathbf{x}_{1: t}, \mathbf{z}_{1: t}\right)$ can become computationally complex and reduce the speed of the filter. A solution to this problem is to use low dimensional sufficient statistics (see [9], [8]) where the parameters depend on $\left(\mathbf{x}_{1: t}, \mathbf{z}_{1: t}\right)$ via a set of conditionally sufficient statistics $s_{t}$ which can be calculated recursively. This implies that there exists a function $\mathcal{S}_{t}$ such that $s_{t}=\mathcal{S}_{t}\left(s_{t-1}, \mathbf{x}_{t}, \mathbf{z}_{t}\right)$ and is initialised as $s_{1}=\mathcal{S}_{1}\left(\mathbf{x}_{1}, \mathbf{z}_{1}\right)$.

\section{Algorithm 1: Particle Learning Filter}

Resample $\left\{\mathbf{x}_{t-1}^{(i)}, \boldsymbol{\theta}^{(i)}\right\}_{i=1}^{N}$
with weights $w_{t}^{(i)} \propto p\left(\mathbf{z}_{t} \mid \boldsymbol{\mu}_{t}^{(i)}, \boldsymbol{\theta}^{(i)}\right)$
where $\boldsymbol{\mu}_{t}^{(i)}$ is given in $(2)$
FOR $i=1, \ldots, N$
Propagate states $\mathbf{x}_{t}^{(i)} \sim p\left(\mathbf{x}_{t} \mid \mathbf{x}_{t-1}^{(i)}, \boldsymbol{\theta}^{(i)}, \mathbf{z}_{t}\right)$
Update sufficient statistics
\[ s_{t}^{(i)}=\mathcal{S}_{t}\left(s_{t-1}^{(i)}, \mathbf{x}_{t}^{(i)}, \mathbf{z}_{t}\right) \]
Sample parameters $\boldsymbol{\theta}^{(i)} \sim p\left(\boldsymbol{\theta} \mid s_{t}^{(i)}\right)$
END

One of the drawbacks of the particle learning approach is that it requires the posterior for the parameters $\boldsymbol{\theta}$ to fit a conditional sufficient statistic structure (i.e. $\left.p\left(\boldsymbol{\theta} \mid \mathbf{x}_{1: t}, \mathbf{z}_{1: t}\right)=p\left(\boldsymbol{\theta} \mid s_{t}\right)\right)$. In situations where this does not hold it is possible to use the Liu and West filter [10] for online parameter estimation.

\subsection{Liu and West Filter}

For certain complex models there does not exist a closed form conjugate prior for the parameters which prevents the use of the particle learning filter. In such situations where there is not a conjugate prior it is possible to perform parameter learning using the Liu and West filter [10]. This filter combines the auxiliary particle filter with a kernel smoothing approximation to estimate the posterior distribution of the parameters using a mixture of multivariate Gaussian densities.

Simple kernel smoothing approximations tend to create an over-dispersed representation of the posterior. This led Liu and West (2001) to include a kernel shrinkage step into the filter which counters the increase in the variance created by the mixture of Gaussian densities. The kernel density form of the smoothed marginal posterior of the parameters is given as

$$
p\left(\boldsymbol{\theta} \mid \mathbf{z}_{1: t}\right) \approx \sum_{i=1}^{N} w_{t}^{(i)} \mathcal{N}\left(\boldsymbol{\theta} \mid \mathbf{m}_{t}^{(i)}, h^{2} \mathbf{V}_{t}\right)
$$

where,

$$
\begin{array}{r}
\mathbf{m}_{t}^{(i)}=a \boldsymbol{\theta}^{(i)}+(1-a) \overline{\boldsymbol{\theta}} \\
\mathbf{V}_{t}=\sum_{i=1}^{N}\left(\boldsymbol{\theta}^{(i)}-\overline{\boldsymbol{\theta}}\right)\left(\boldsymbol{\theta}^{(i)}-\overline{\boldsymbol{\theta}}\right)^{T} / N
\end{array}
$$

with $\overline{\boldsymbol{\theta}}=\sum_{i=1}^{N} \boldsymbol{\theta}^{(i)} / N$ and $a=\sqrt{1-h^{2}}$ where $h>$ 0 is the smoothing parameter.

\section{Algorithm 2: Liu and West Filter}

Resample $\left\{\mathbf{x}_{t-1}^{(i)}, \boldsymbol{\theta}^{(i)}\right\}_{i=1}^{N}$
with weights $w_{t} \propto w_{t-1}^{(i)} p\left(\mathbf{z}_{t} \mid \boldsymbol{\mu}_{t}^{(i)}, \mathbf{m}_{t-1}^{(i)}\right)$
where $\boldsymbol{\mu}_{t}$ is given in $(2)$ and $\mathbf{m}_{t-1}^{(i)}$ is given in (3)
FOR $i=1, \ldots, N$
$\quad$ Update parameters $\boldsymbol{\theta}^{(i)} \sim \mathcal{N}\left(\boldsymbol{\theta} \mid \mathbf{m}_{t-1}^{(i)}, h^{2} \mathbf{V}_{t-1}\right)$
$\quad$ where $\mathbf{V}_{t-1}$ is given in $(4)$
$\quad$ Propagate states $\mathbf{x}_{t}^{(i)} \propto p\left(\mathbf{x}_{t} \mid \mathbf{x}_{t-1}^{(i)}, \boldsymbol{\theta}^{(i)}\right)$
$\quad$ Assign weights $w_{t}^{(i)} \propto \frac{p\left(\mathbf{z}_{t} \mid \mathbf{x}_{t}^{(i)}, \boldsymbol{\theta}^{(i)}\right)}{p\left(\mathbf{z}_{t} \mid \boldsymbol{\mu}_{t}^{(i)}, \mathbf{m}_{t-1}^{(i)}\right)}$
END

\subsection{Particle Learning with Change- points}

Parameter estimation filters such as the particle learning filter and Liu and West filter are constrained to parameter learning for static parameters. In target tracking, there exist scenarios where changes in the motion of the target require the parameters of the model to evolve in conjunction with the target's motion. Previous approaches to this problem have involved modelling the target with multiple models as in the IMM filter. However, these techniques do not learn about the parameters of interest but compensate by switching between various models with different parameter values.

This filter extends upon the approaches to online estimation for static parameters to allow for piecewise time-varying parameters. We assume that there exist $m$ changepoints at unknown time points $\tau_{1: m}=\left\{\tau_{1}, \tau_{2}, \ldots, \tau_{m}\right\}$ in the observations such that $p\left(\mathbf{z}_{1: \tau_{i}}, \boldsymbol{\theta}_{1: \tau_{i}}\right) \neq p\left(\mathbf{z}_{\tau_{i}+1: t}, \boldsymbol{\theta}_{\tau_{i}+1: t}\right)$, which segments the observations at times $\tau_{i}$. The probability of a changepoint at $\tau_{i}$ is given as $\beta$, which we assume is fixed throughout time. Using this changepoint method, it is now possible to assume that the parameters $\boldsymbol{\theta}_{t}$ within a segment are static and can be estimated using filters such as those discussed. However, once the observations of the target indicate that the target has performed a maneuver, the filter will identify the maneuver as a changepoint and will update the parameters by drawing new parameter values from the initial prior distribution of the parameters to begin learning the new parameter values.

This approach also follows from the auxiliary particle filter, in the sense that the first step is 
Algorithm 3: Particle Learning with Changepoints

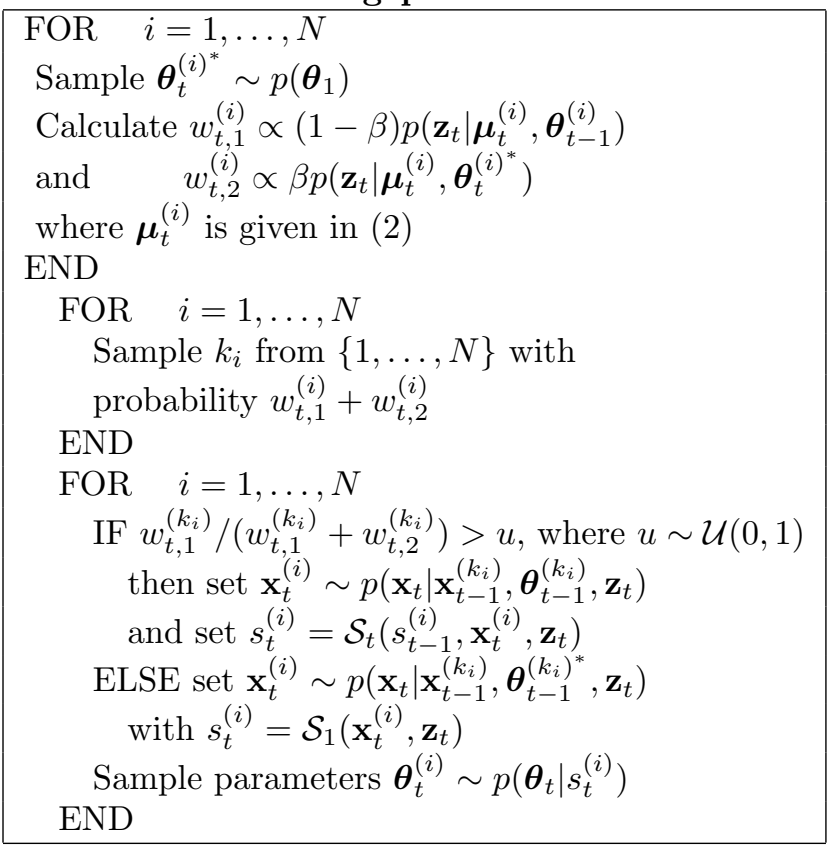

to resample the particles with weights proportional to the predictive likelihood. This approach differs from the previous methods in that the predictive likelihood is calculated given the learnt parameters $w_{t, 1}^{(i)} \propto p\left(\mathbf{z}_{t} \mid \boldsymbol{\mu}_{t}^{(i)}, \boldsymbol{\theta}_{t-1}^{(i)}\right)$ and the fresh parameters $w_{t, 2}^{(i)} \propto p\left(\mathbf{z}_{t} \mid \boldsymbol{\mu}_{t}^{(i)}, \boldsymbol{\theta}_{t}^{(i)^{*}}\right)$, which are parameters drawn from the initial prior. All particles are then resampled given a resampling scheme such as multinomial or stratified sampling. The resampling weights are given as $w_{t, 1}^{(i)} \propto(1-\beta) p\left(\mathbf{z}_{t} \mid \boldsymbol{\mu}_{t}^{(i)}, \boldsymbol{\theta}_{t-1}^{(i)}\right)$ and $w_{t, 2}^{(i)} \propto \beta p\left(\mathbf{z}_{t} \mid \boldsymbol{\mu}_{t}^{(i)}, \boldsymbol{\theta}_{t}^{(i)^{*}}\right)$, where $\beta$ is the probability of a changepoint. This approach can be utilised as an extension to both the particle learning filter (Algorithm 3) and Liu and West filter (Algorithm 4) depending on whether there exist closed form conjugate priors for the parameters.

\section{Simulation Study}

In this scenario the target moves within the $x y$ plane, where the target's state vector is $\mathbf{x}_{\mathbf{t}}=$ $\left(x_{t}, \dot{x}_{t}, y_{t}, \dot{y}_{t}\right)^{\prime}$. The motion of the target is modelled using a coordinated-turn model [11] of the form

$$
\mathbf{x}_{\mathbf{t}}=\mathbf{F} \mathbf{x}_{\mathbf{t}-\mathbf{1}}+\boldsymbol{\nu}_{t}
$$

where,

$$
\mathbf{F}=\left[\begin{array}{cccc}
1 & \frac{\sin \omega_{t} T}{\omega_{t}} & 0 & -\frac{1-\cos \omega_{t} T}{\omega_{t}} \\
0 & \cos \omega_{t} T & 0 & -\sin \omega_{t} T \\
0 & \frac{1-\cos \omega_{t} T}{\omega_{t}} & 1 & \frac{\sin \omega_{t} T}{\omega_{t}} \\
0 & \sin \omega_{t} T & 0 & \cos \omega_{t} T
\end{array}\right]
$$

and $\omega_{t} \in(-\pi \mathrm{rads} / \mathrm{s}, \pi \mathrm{rads} / \mathrm{s})$ is the rate of turn and $T=1 s$ is the sampling time given in seconds.
Algorithm 4: Liu and West Filter with Changepoints

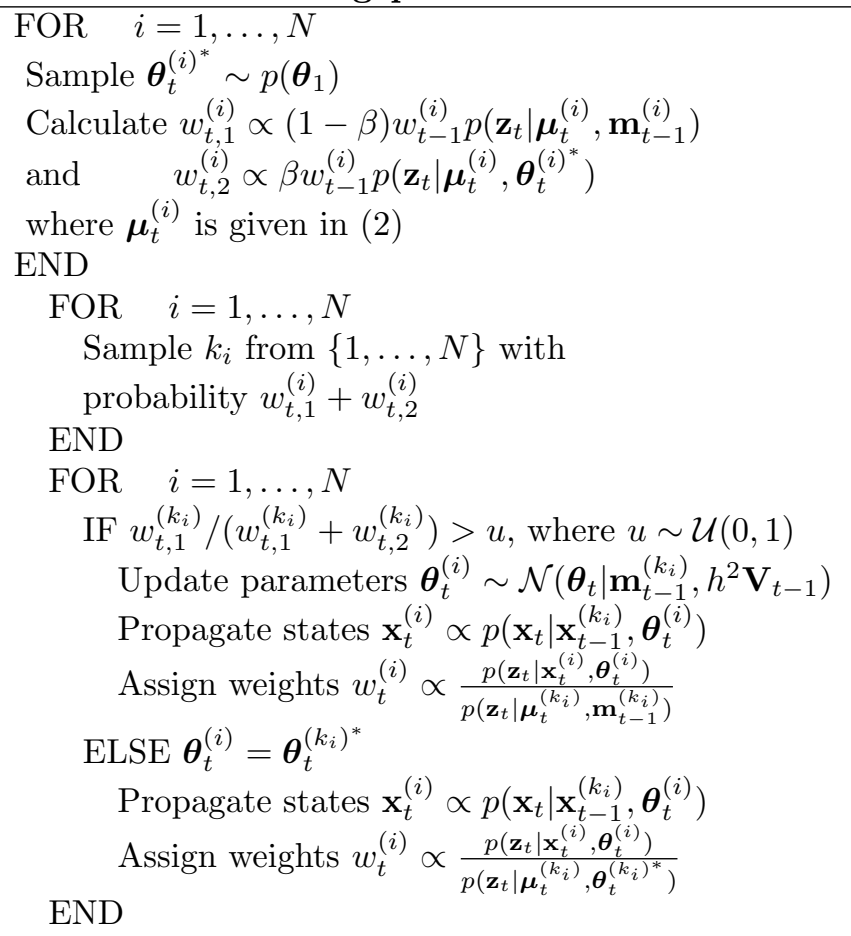

The system noise $\boldsymbol{\nu}_{t}$ is given as a zero mean Gaussian white noise processes with known covariance $\mathbf{Q}=\operatorname{diag}(2 m, 1 \mathrm{~m} / \mathrm{s}, 2 \mathrm{~m}, 1 \mathrm{~m} / \mathrm{s})$. In the limiting case of the rate of turn parameter $\omega_{t} \rightarrow 0$ the coordinated turn model simplifies to the constant velocity model [12] or the form

$$
\mathbf{F}=\left[\begin{array}{cccc}
1 & T & 0 & 0 \\
0 & 1 & 0 & 0 \\
0 & 0 & 1 & T \\
0 & 0 & 0 & 1
\end{array}\right]
$$

which shall be used in the scenario to model nearly constant target velocity.

Noisy observations of the target are taken by a fixed observer positioned at $\left(s_{x}, s_{y}\right)$, with the range and bearing of the target are given as

$$
\mathbf{z}_{\mathbf{t}}=\left[\begin{array}{c}
\sqrt{\left(x_{t}-s_{x}\right)^{2}+\left(y_{t}-s_{y}\right)^{2}} \\
\arctan \left(\left(y_{t}-s_{y}\right) /\left(x_{t}-s_{x}\right)\right)
\end{array}\right]+\boldsymbol{\epsilon}_{t}
$$

where the observation noise $\boldsymbol{\epsilon}_{t}$ is a zero mean Gaussian white noise process with known covariance $\mathbf{R}=\operatorname{diag}(10 m, 0.001 \mathrm{rads})$. The initial state of the target is $\mathbf{x}_{1}=(0 \mathrm{~m}, 10 \mathrm{~m} / \mathrm{s}, 0 \mathrm{~m}, 10 \mathrm{~m} / \mathrm{s})^{\prime}$ and the observer is positioned at $(150,-50)$.

In this scenario the aim is to apply the new filter, the Liu and West changepoint filter, against the IMM filter and original Liu and West filter, where it is assumed that the turn rate parameter $\omega_{t}$ is unknown and changes at some unknown point in time. The simulated target track has been created from the coordinated turn model over 80 time 
steps, where the turn rate parameter $\omega_{t}$ takes values $(0,-\pi / 20,0, \pi / 20,0)$ at times $(1-19,20-39,40-$ $59,60-80)$, respectively. See Figure 1 for the simulated scenario.

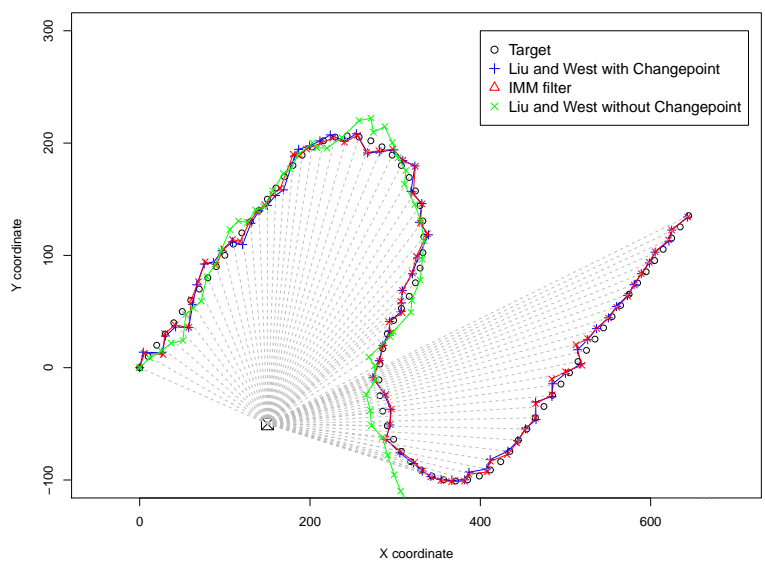

Figure 1: Simulated target path

The Liu and West changepoint filter is implemented with 10,000 particles and smoothing parameter $h^{2}=0.1$ as recommended by Liu and West [10], where the probability of a changepoint at any point in time is $\beta=0.05$. The prior distribution for the turn rate parameter $\omega_{t}$ follows a uniform distribution over the range $[-\pi / 8, \pi / 8]$. The IMM filter is a robust alternative to filters which estimate the parameters of interest in real-time. Rather than attempting to estimate the parameter the IMM is implemented with multiple models, where at each time step an estimate of the target's position is given by weighting and merging the estimates of the individual models. In this scenario the IMM filter will be implemented using 20 coordinated turn models. The models will differ only in the choice of the parameter $\omega_{t}$, where 20 equally spaced values of $\omega_{t}$ are sampled over the range $[-\pi / 8, \pi / 8]$. The transition probabilities between models of the IMM filter are balanced equally between all alternative models and sum to 0.05 with a 0.95 probability of no model transition. As the observation model is nonlinear the IMM filter is to be implemented with an unscented Kalman filter.

From Figure 1 it can be seen that both the Liu and West changepoint filter and the IMM filter are able to track the target with reasonable accuracy. However, the original Liu and West filter without a changepoint step, which performs parameter estimation of static parameters, struggles to track the target. Initially, this filter appears to estimate the target's position well and quickly learns that the unknown parameter $\omega_{t}$ is zero. However, once the target performs the first maneuver the error in the estimate of the target's position given by the Liu and West filter increases. The increase in error is caused by the learnt parameter, which no longer matches the parameter describing the target's motion. The error in the estimate given by the filter increases as the filter attempts to track the target with a mis-specified parameter, after the second maneuver the filter loses track of the target completely.

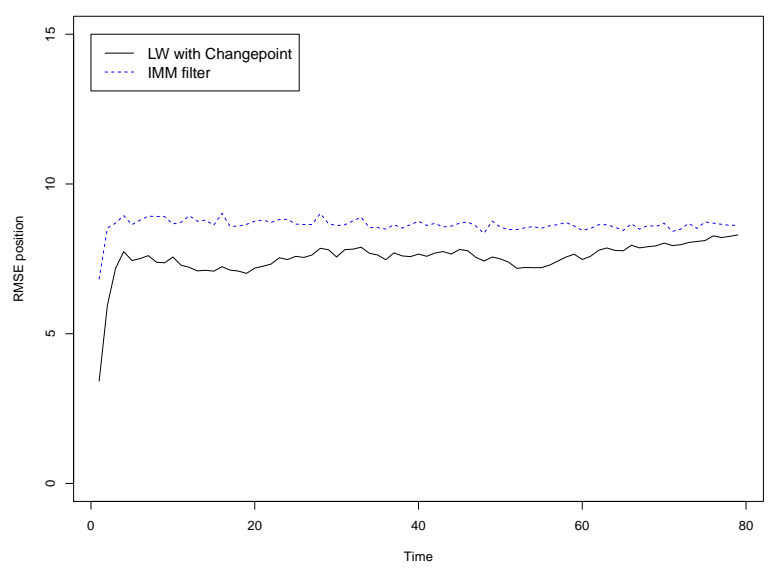

Figure 2: Root mean squared position error of target estimate

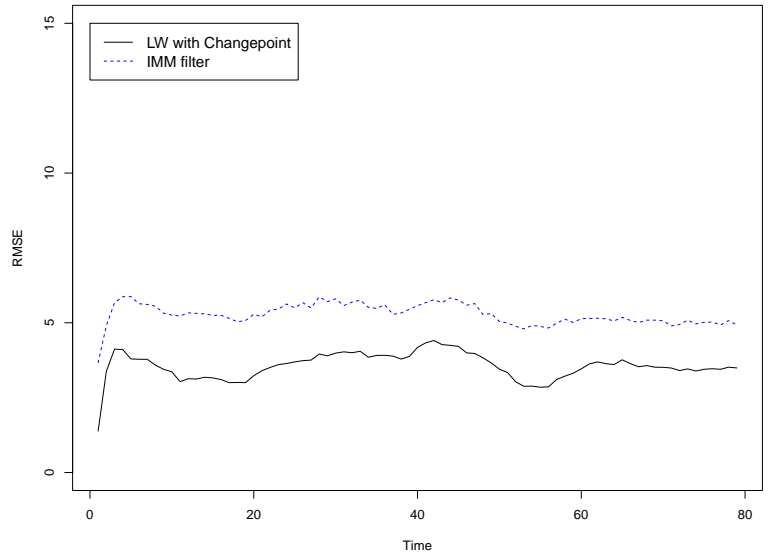

Figure 3: Root mean squared velocity error of target estimate in $y$ axis

The performance of the Liu and West changepoint filter and the IMM filter is measured using the root mean squared error (RMSE) of the target's true position against its estimated position (Fig. 2) over 2000 Monte Carlo runs. From the RMSE it can be seen that for the IMM filter and Liu and West changepoint filter show similar levels accuracy. However, over the 80 time steps the Liu and West changepoint filter has a lower RMSE than the IMM filter. A similar result follows for the velocity of the target (Fig. 3) which is nearly constant during the non-maneuvering phases. Changes in the velocity occur when the target turns (time steps 20, 40 and 
60 ), there is also a corresponding small increase in the RMSE for the velocity during these periods.

\section{Conclusions}

The Liu and West changepoint filter performs well against the traditional IMM filter in the case of tracking a target with nonlinear observations. Standard parameter estimation filters such as the Liu and West filter work well in estimating the values of the parameters of interest, but do not permit changes in parameter values. The flexibility of the IMM filter allows targets which can be highly maneuverable to be tracked, but as the parameters of the various models are fixed the IMM filter can struggle to track a target if the models implemented within the filter are not correctly specified. Alternatively, if there are multiple unknown parameters then the IMM filter would need to be implemented with a greater combination of models in order to account for the various changes in the target's behaviour. Overall, the Liu and West changepoint model appears to perform equally well to the IMM filter when the parameters are static, but has an advantage over the IMM filter during in periods of parameter uncertainty.

\section{Acknowledgments}

The authors would like to acknowledge the support from EPSRC (grant EP/G501513/1) and MBDA, UK.

\section{References}

[1] C. Andrieu, a. Doucet, S. Singh, and V. Tadic, "Particle Methods for Change Detection, System Identification, and Control," Proceedings of the IEEE, vol. 92, no. 3, pp. 423-438, Mar. 2004.

[2] H. Blom and Y. Bar-Shalom, "The interacting multiple model algorithm for systems with markovian switching coefficients," IEEE Transactions on Automatic Control, vol. 33, no. 8, pp. $780-783$, Aug 1988.

[3] C. Carvalho, M. Johannes, H. Lopes, and N. Polson, "Particle Learning and Smoothing," Statistical Science, vol. 25, no. 1, pp. 88-106, 2010.

[4] H. Lopes and R. Tsay, "Particle filters and Bayesian inference in financial econometrics," Journal of Forecasting, vol. 30, no. 1, pp. 168209, 2011.
[5] N. Gordon, D. Salmond, and A. Smith, "Novel approach to nonlinear and linear Bayesian state estimation," IEE Proceedings, vol. 140, no. 2, pp. 107-113, 1993.

[6] L. Mihaylova and D. Angelova, "Noise Parameters Estimation with Gibbs Sampling for Localisation of Mobile Nodes in Wireless Networks," in Proceedings of the 13th International Conference on Information Fusion, Edinburgh, UK, 2010.

[7] M. K. Pitt and N. Shephard, "Filtering via Simulation: Auxiliary Particle Filters," Journal of the American Statistical Association, vol. 94, no. 446, pp. 590-599, June 1999.

[8] G. Storvik, "Particle filters for state-space models with the presence of unknown static parameters," IEEE Transactions on Signal Processing, vol. 50, no. 2, pp. 281-289, 2002.

[9] P. Fearnhead, "Markov chain Monte Carlo, Sufficient Statistics, and Particle Filters," Journal of Computational and Graphical Statistics, vol. 11, no. 4, pp. 848-862, Dec. 2002.

[10] M. West and J. Liu, "Combined parameter and state estimation in simulation-based filtering," in Sequential Monte Carlo Methods in Practice, A. Doucet, N. de Freitas, and N. Gordon, Eds. New York: Springer-Verlag, 2001, pp. 197-223.

[11] X. Rong Li and Y. Bar-Shalom, "Design of an Interacting Multiple Model Algorithm for Air Traffic Control Tracking," IEEE Transactions on Control Systems Technology, vol. 1, no. 3, pp. 186-194, 1993.

[12] Y. Bar-Shalom, X. R. Li, and T. Kirubarajan, Estimation with Applications to Tracking and Navigation. John Wiley \& Sons, 2001. 